3. Galés C, Rebois RV, Hogue M, et al. Real-time monitoring of receptor and $\mathrm{G}$-protein interactions in living cells. Nat Methods $2005 ; 2$ : 177-84.

4. Pfleger KD, Eidne KA. Illuminating insights into protein-protein interactions using bioluminescence resonance energy transfer (BRET). Nat Methods 2006 ; 3: 165-74.

5. Galés C, Van Durm JJ, Schaak S, et al. Probing the activation-promoted structural rearrangements in preassembled receptor- $G$ protein complexes. Nat Struct Mol Biol $2006 ; 13$ : 778-86.
6. Cherfils J, Chabre M. Activation of G-protein Galpha subunits by receptors through Galpha-Gbeta and Galpha-Ggamma interactions. Trends Biochem Sci $2003 ; 28: 13-7$

7. Bourne HR. How receptors talk to trimeric $\mathrm{G}$ proteins. Curr Opin Cell Biol 1997 ; $9: 134-42$.

8. Lavine N, Éthier N, Oak JN, et al. G protein-coupled receptors form stable complexes with inwardly rectifying potassium channels and adenylyl cyclase. J Biol Chem 2002 ; 277 : 46010-9.
9. Riven I, Iwanir S, Reuveny $\varepsilon$. GIRK channel activation involves a local rearrangement of a preformed $G$ protein channel complex. Neuron 2006 ; 51 : 561-73.

10. Rebois RV, Robitaille M, Galés C, et al. Heterotrimeric $G$ proteins form stable complexes with adenylyl cyclase and Kir3.1 channels in living cells. J Cell Sci 2006 ; 119 : 2807-18.

\title{
NOUVELle
}

\section{PGC- $1 \alpha$ régule la jonction neuromusculaire et améliore la dystrophie musculaire de Duchenne}

Anne-Sophie Arnold
Physiology Institute, Université de Zürich, Winterthurerstrasse 190, CH-8057 Zürich, Suisse. a.arnold@access.uzh.ch
$>$ Le coactivateur $l \alpha$ du récepteur $\gamma$ activé par le proliférateur du peroxisome (PPAR $\gamma$ ), ou PGC-l $\alpha$, identifié par l'équipe de Christoph Handschin et Bruce Spiegelman, confère une spécificité transcriptionnelle à PPAR $\gamma[1]$. Ce facteur est en fait une plateforme protéique qui relie les facteurs de transcription au complexe d'initiation de la transcription et participe au traitement de I'ARN messager cible.

La fonction principale de PGC-l $\alpha$ est de stimuler l'augmentation du nombre de mitochondries ainsi que le métabolisme oxydatif. Ce facteur est en effet très exprimé dans les tissus caractérisés par un métabolisme oxydatif intense, comme le cœur, le rein, le cerveau, le tissu adipeux brun, et le muscle squelettique [2]. C'est en particulier au niveau de ce dernier tissu que PGC- $1 \alpha$ semble jouer un rôle très important. La concentration en PGC-l $\alpha$ est plus forte dans les muscles riches en fibres de type I et II a, comme le muscle soléaire [3], et l'expression de PGC-l $\alpha$ est favorisée par l'exercice, à la fois chronique et aigu [4]. II a été suggéré que ce facteur pourrait être le médiateur des effets antiapoptotiques qu'exerce l'activité nerveuse sur la masse musculaire.

\section{PGC-1 $\alpha$}

et la jonction neuro-musculaire

Ainsi, PGC- $1 \alpha$ semble être un régulateur important dans le programme de la jonction neuromusculaire (JNM). La taille et la morphologie de la JNM évoluent pendant le développement, mais également selon le degré d'activité physique ainsi que la distribution des différents types de fibres musculaires présents. D'après Handschin et al., PGC-l $\alpha$ est très important dans le cadre de l'adaptation du muscle aux changements d'activité (exercice, modifications physiologiques), mais il n'a qu'un rôle modéré dans la régulation des fonctions musculaires de base. Les niveaux d'expression de PGC-l $\alpha$ sont également importants pour la formation des agrégats de récepteurs à l'acétylcholine dans les fibres musculaires isolées, une des étapes précoces de l'installation de la JNM.

Les voies de signalisation de l'agrine et de la neuréguline 1 (NRG-1), deux acteurs essentiels dans la formation et la plasticité de la JNM, convergent toutes les deux vers le facteur de transcription GA binding protein (GABP), dont l'activité est régulée par PGC-l $\alpha$ $[5,6]$. Pour la première fois, il est montré que PGC-l $\alpha$ établit une réelle liaison physique avec GABP et la coactive. En fait, PGC-l $\alpha$ est nécessaire pour que la phosphorylation de GABP activée par NRG-1 (et par conséquent l'augmentation d'expression des gènes de la JNM) ait lieu. Cette interaction entre PGC- $1 \alpha$ et GABP dépend étroitement de leur état de phosphorylation respectif. Les auteurs suggèrent que les modifications post-traductionnelles de GABP sont responsables du recrutement de PGC-l $\alpha$ par GABP.

\section{Action bénéfique} de PGC- $1 \alpha$ dans les myopathies

Les auteurs de cette étude ont fait des observations très intéressantes chez des souris $m d x$ (le modèle animal classique pour la myopathie musculaire de Duchenne ou DMD) transgéniques pour l'expression ciblée de PGC-l $\alpha$ uniquement dans le muscle. II en résulte une amélioration nette du phénotype de ces souris qui se traduit par des niveaux plus bas de créatine kinase, une réduction du nombre de lésions nécrotiques, une moindre altération des fibres musculaires, et une amélioration des fonctions musculaires 


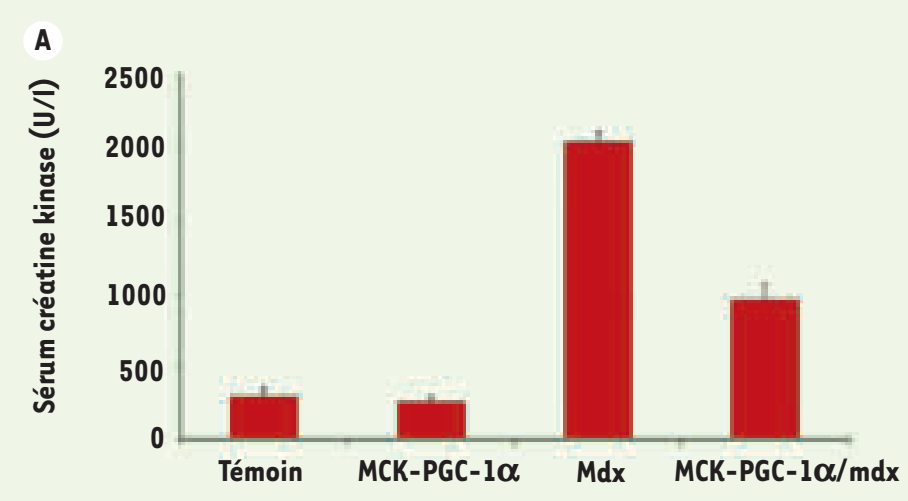

B
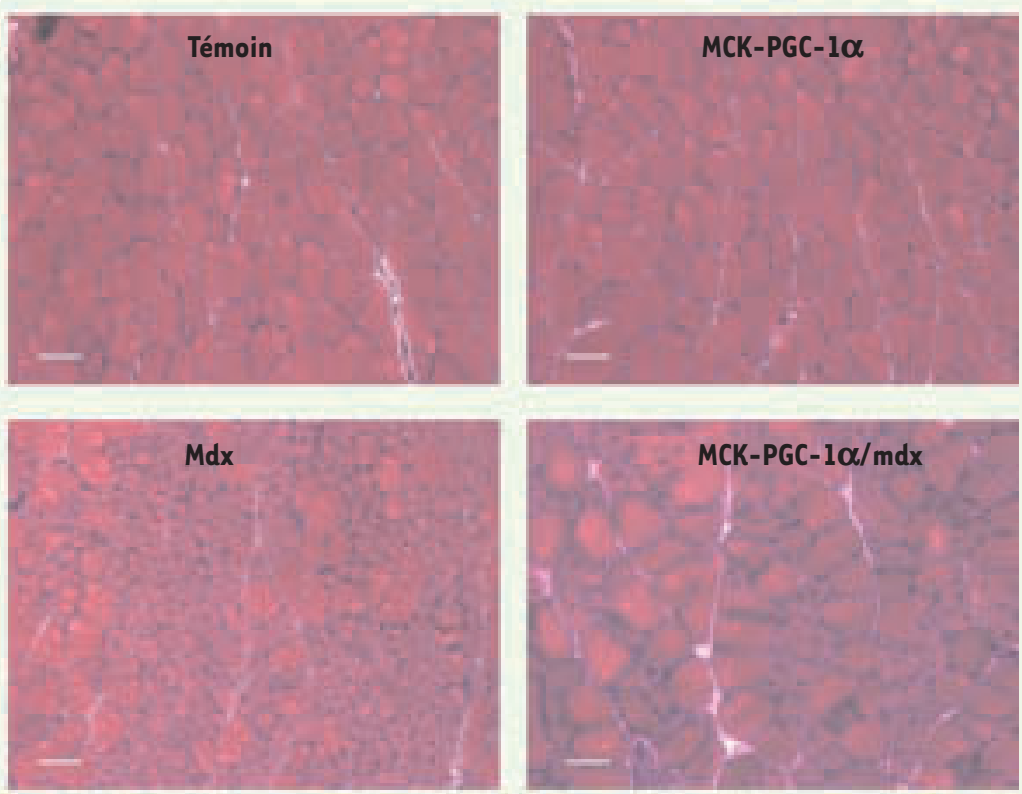

C
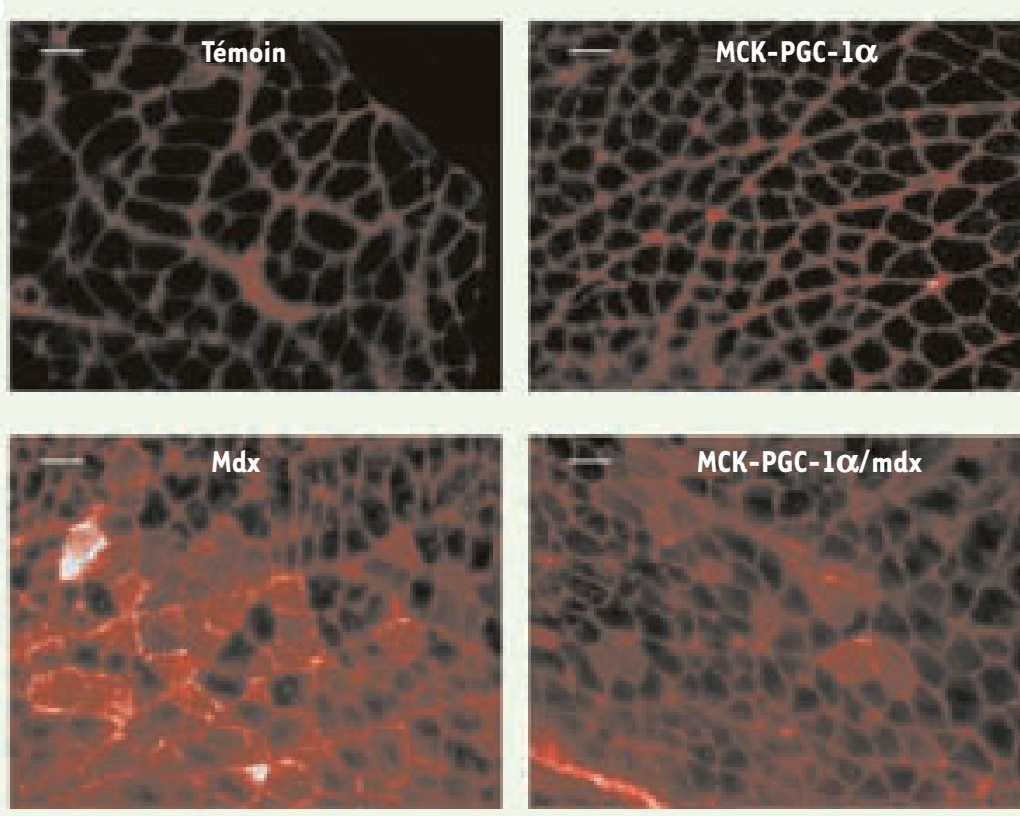

(Figure 1). Cet allègement de la dystrophie musculaire par PGC-l $\alpha$ chez les souris $m d x$ requiert l'induction d'expression des transcrits et de la protéine utrophine. L'utrophine est depuis longtemps un candidat thérapeutique potentiel très sérieux pour se substituer à l'absence de dytrophine chez les patients DMD [7].

Mais les effets bénéfiques de PGC-l $\alpha$ vont très au-delà de la simple stimulation de la synthèse d'utrophine. (1) PGC- $1 \alpha$ a un effet anti-apoptotique sur les fibres musculaires après dénervation (nerf sciatique). Cet effet passe, entre autres, par l'inhibition du facteur pro-apoptotique Fox03 [8]. (2) PGC- $1 \alpha$ est un régulateur puissant de la biogenèse des mitochondries et $d u$ métabolisme oxydatif dans le muscle squelettique. II est bien connu que les muscles squelettiques des souris $m d x$ présentent des défauts au niveau de la phosphorylation oxydative et des fonctions mitochondriales. (3) PGC$1 \alpha$ est une cible directe de la voie calcium/calmoduline dans le muscle squelettique. Or, l'interruption de cette voie dans les souris $m d x$ exacerbe le

Figure 1. PGC-1 $\alpha$ améliore la dystrophie musculaire chez les souris $\mathrm{mdx}$ sédentaires. Les souris utilisées dans les expériences décrites dans cette figure sont des souris témoins âgées de 5 semaines, des souris transgéniques pour PGC-l $\alpha$ uniquement dans le muscle (MCK$P G(-1)$, des souris $m d x$, ainsi que des souris $m d x$ transgéniques pour $\mathrm{PGC}-1 \alpha$ uniquement dans le muscle (MCK-PGC-1/mdx) (A). Les histogrammes représentent le niveau moyen de créatine kinase et les barres d'erreur représentent la déviation standard. B. Histologie des muscles gastrocnémiens après coloration à I'hématoxyline-éosine. C. Une solution de bleu Evans à $1 \%$ est injectée en intrapéritonéal et les souris sont sacrifiées $16 \mathrm{~h}$ plus tard. L'incorporation du colorant par les fibres musculaires est observée par microscopie fluorescente (Reproduit de [1] avec I'autorisation de Genes \& Development, Cold Spring Harbor Laboratory Press, 2007 (C). 
phénotype dystrophique [9]. (4) L'effet qu'exerce PGC-l $\alpha$ sur les gènes de la JNM pourrait minimiser les défauts de morphologie de la JNM observés chez les souris $m d x$ lors de la régénération de la fibre musculaire après une blessure. Enfin, la production de reactive oxygen species (ROS) pourrait être à l'origine des dommages musculaires observés chez la souris $m d x$. Or, pour se défendre contre la toxicité de ces espèces, l'organisme fait appel à un régulateur puissant des programmes génétiques, la protéine $\mathrm{PGC}-1 \alpha$.

\section{Conclusions}

Pour résumer, l'exposé des résultats cités ci-dessus prouve indubitablement que ce facteur PGC-l $\alpha$ ouvre la voie pour le développement de nouvelles cibles thérapeutiques dans le cadre de la DMD, ou de sa forme légère, la dystrophie musculaire de Becker, mais aussi pour d'autres maladies neuromusculaires. $\diamond$

PGC- $1 \alpha$ controls neuromuscular junction and offers a novel therapeutic target in Duchenne dystrophy?

\section{RÉFÉRENCES}

1. Handschin C, Kobayashi YM, Chin S, et al. PGC-lalpha regulates the neuromuscular junction program and ameliorates Duchenne muscular dystrophy. Genes Dev $2007 ; 21: 770-83$

2. Handschin C, Spiegelman BM. Peroxisome proliferator-activated receptor gamma coactivator 1 coactivators, energy homeostasis, and metabolism. Endocr Rev 2006; 27 : 728-35.

3. Lin J, Wu H, Tarr PT, Zhang CY, et al. Transcriptional coactivator PGC-1 alpha drives the formation of slowtwitch muscle fibres. Nature $2002 ; 418: 797-801$.

\section{NOUVELlE}

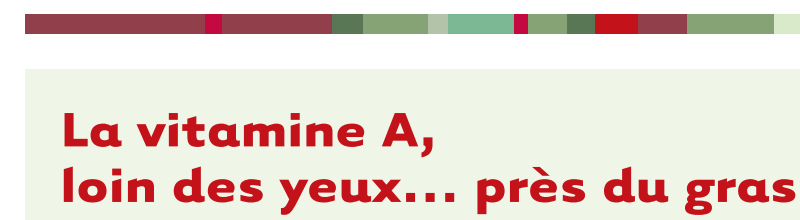

Isabelle Dugail, Pascal Ferré

\section{Métabolisme de la vitamine A}

La vitamine $A$, ou rétinol, est un composé liposoluble apporté par l'alimentation, abondant dans le lait, les œufs, le foie et les huiles de poisson. Certains végétaux riches en $\beta$-carotènes (ou provitamine $A$ ) sont aussi des sources abondantes, mais une étape d'activation est alors nécessaire pour obtenir la vitamine. Des rôles multiples et complexes ont été décrits pour la vitamine $A$, dans la croissance cellulaire, la différenciation et la reproduction, mais son effet biologique «phare» (si l'on peut dire!) s'exerce au niveau de la rétine. La vitamine $A$ est transformée en rétinal. Le rétinal s'associe à une protéine, l'opsine, pour former la rhodopsine présente dans les cellules photoréceptrices. Sous l'effet des photons, la structure du rétinal est modifiée et la rhodopsine change de conforma- tion, ce qui induit, après un certain nombre d'étapes, le signal nerveux rétinien. Les carences en vitamine $A$, fréquentes en cas de malnutrition, sont une cause majeure de cécité dans les pays les plus pauvres.

Le rétinol, hydrophobe, circule dans le compartiment sanguin conjugué à des protéines de transport comme les retinol binding protein ( $\mathrm{RBP})$. Au niveau intracellulaire, le rétinol est transformé en rétinaldéhyde par une alcool déshydrogénase puis en acide rétinoïque par la rétinaldéhyde déshydrogénase de type 1 (raldhl) [1]. Les différents isomères de l'acide rétinoïque (9-cis et tout-trans) peuvent réguler la transcription de nombreux gènes car ils sont des ligands naturels des récepteurs nucléaires de la famille RAR (retinoic acid receptor) et $\mathrm{RXR}$ (retinoid $X$ recep-
4. Russell AP, Feilchenfeldt J, Schreiber S, et al. Endurance training in humans leads to fiber typespecific increases in levels of peroxisome proliferatoractivated receptor-gamma coactivator-l and peroxisome proliferator-activated receptor-alpha in skeletal muscle. Diabetes 2003; 52 : 2874-81.

5. Angus LM, Chakkalakal JV, Mejat A, et al. CalcineurinNFAT signaling, together with GABP and peroxisome PGC-1\{alpha\}, drives utrophin gene expression at the neuromuscular junction. Am J Physiol Cell Physiol $2005 ; 289$ : C908-17.

6. Briguet $A$, Ruegg MA. The Ets transcription factor GABP is required for postsynaptic differentiation in vivo. J Neurosci $2000 ; 20: 5989-96$.

7. Miura P, Jasmin BJ. Utrophin upregulation for treating Duchenne or Becker muscular dystrophy: how close are we? Trends Mol Med 2006 ; 12 : 122-9.

8. Sandri M, Lin J, Handschin C, Yang W, et al. PGClalpha protects skeletal muscle from atrophy by suppressing Fox03 action and atrophy-specific gene transcription. Proc Natl Acad Sci USA 2006 ; 103: 16260-5.

9. Handschin C, Rhee J, Lin J, et al. An autoregulatory loop controls peroxisome proliferator-activated receptor gamma coactivator lalpha expression in muscle. Proc Natl Acad Sci USA 2003 ; 100 : 7111-6.
Inserm, U872, Paris, F-75006 France ; Centre de Recherche des Cordeliers, Université Pierre et Marie Curie - Paris 6, UMR S872, Paris, F-75006 France ; Université Paris Descartes, UMR S 872 , Paris, F-75006 France.

Centre de recherche des cordeliers, Équipe 8, 15, rue de l'École de Médecine, 75006 Paris, France.

isabelle.dugail@crc.jussieu.fr pascal.ferre@crc.jussieu.fr

tor). Le rétinol est stocké dans le foie mais également dans la gouttelette lipidique de l'adipocyte, sous forme d'esters.

\section{Rétinaldéhyde}

et développement du tissu adipeux

Un article publié en mai 2007 dans la revue Nature Medicine [2] démontre que le rétinaldéhyde exerce des effets spécifiques dans le développement du tissu adipeux et dans la régulation de l'homéostasie glucido-lipidique, distincts de ceux des isomères de l'acide rétinoïque. Les auteurs ont observé la présence de concentrations importantes de rétinal- 